\title{
Synthetizing State Feedback Control Laws for Discrete Event Systems with Constrained Paths Using Min-Plus Algebra
}

\author{
Nesrine BEN AFIA ${ }^{1 *}$, Said AMARI ${ }^{2}$ \\ ${ }^{1}$ LARATSI, National Engineering School of Monastir, 5019, Tunisia \\ nesrine.benafia@enim.u-monastir.tn (*Corresponding author) \\ ${ }^{2}$ LURPA, Normal Superior School of Paris-Saclay, 91190, France \\ samari@ens-paris-saclay.fr
}

\begin{abstract}
This paper outlines a new algebraic method for controlling Timed Event Graphs to satisfy upper time bounds imposed on certain critical paths. Adequate conditions that pave the way for the existence of control laws in the form of state feedbacks have been established by relaxing and generalizing some previous works. The aim is to ensure compliance of these strict time constraints while keeping the main properties of the Timed Event Graphs such as liveness. The resulting control laws correspond to a set of added monitor places preventing the violation of time constraints. The theoretical results were applied to a manufacturing cell as a part of a flexible workshop exposed to strict time duration within some of its processes.
\end{abstract}

Keywords: Discrete Event Systems (DES), Timed Event Graphs (TEG), Min-Plus algebra, Temporal constraints, Control synthesis.

\section{Introduction}

This paper deals with the control synthesis problem of Discrete Event Systems under strict time path constraints. In such systems with a crucial period, the respect of the temporal constraints proves its capability in the evolution of the real industrial processes. Therefore, the efficiency of these kinds of systems depends not only on their logical accuracy but also on their compliance with time specifications. Overall, constraints are involved in various aspects such as deadline, time intervals, validity duration, desired response time ... and so on. They are often integrated in various real applications like: flexible manufacturing workshops including thermal or chemical treatments; as in (Mhalla et al., 2013) and in (Kim \& Lee, 2016), real-time systems which are the object of the research conducted by Dasarathy (1985) and urban and rail transports evoked in (Holloway et al., 1997), (Houssin et al., 2007), and (Wang et al., 2013). Networked automation systems were investigated in the work of (Addad et al., 2010) and (Tebani et al., 2017). In the specialized literature, a variety of study cases and works have focused their attention on the problem of the control synthesis approaches related to time-constrained systems, take the example of (Tiberiu et al., 2006) and (Sava \& Alla, 2007). Different resolution methods have been proposed in turn.

A class of Discrete Event Systems involving tasks with real-time constraints is tackled in (Mao \& Cassandras, 2009) and (Mao \& Cassandras, 2010).
The main purpose beyond of the developed approaches is to control processing times as well as to minimize a cost function subject to each task meeting its own constraint. The author Bonhomme $(2001,2013)$ has developed a control approach for real-time systems modeled by a class of Petri nets. The results of (Lahaye et al., 2004) and (Cottenceau et al., 1999) involve some resolution techniques related to disturbance rejection and tracking model issues. Similarly, to avoid any violation of time specification, a technique of regulating wafer delays against timing disruptions is in the mean and it is established in the contribution of (Kim \& Lee, 2016). In the same process, after managing to model the behavior of a tool by a Timed Event Graph, they developed a feedback controller for single-armed and dual-armed cluster tools that can satisfy the time constraints by regulating wafer delays. The work of (Houssin et al., 2013) has restricted the treatment of time constraints only on some particular parts of the Timed Event Graphs. In doing so, they assumed that the first transitions of these constrained paths are directly controllable (i.e. these transitions are connected by a single place with input transitions). Within the same context, Houssin et al. (2007) managed to find other control strategies that were particularly integral to the just-in-time criterion for constrained $(\max ,+)$ linear systems. The proposed approach was applied to the timetables 
synthesis of urban bus networks. In (Wang et al., 2013) and with similar application context, under constraints and fixed arrival time, a pseudospectral method and a mixed integer linear programming approach are combined to solve an optimal trajectory planning problem for train operations. Other methods are also developed to design a controller to ensure that a MaxPlus system evolves without violating the time restrictions imposed on the state; were addressed in (Maia et al., 2013) or even characterized by a semimodule (Maia et al., 2011b). Other research achievements related to the control problem like also in (Maia et al., 2011a), where the approach was essentially based on the algebraic properties of the system matrices by solving linear MaxPlus equation and by Checking sufficient conditions. In addition, the system's stability was also an object of study.

Dealing with this kind of control problem has required the emergence of a variety of research activities as presented in Amari (2005) and (Jacob \& Amari, 2017). To solve the feedback control problem of Timed Event Graphs under strict time constraints, authors have developed several methodologies using Max-Plus or MinPlus algebra. Dealing with the same topic, it has been showed in the achieved results of (Tebani et al., 2018) that control laws may exist under the assumption of an empty path between the control transition and the upstream transition of the constrained place. Consequently, this specific condition on the empty path remains risky due to the capability of introducing loop without a token (i.e. when the paths are closed, the system loses its liveness property).

Since the previous approaches showed a remarkable limitation (all markings of the constrained places are null besides to the existence of an empty path between the control transitions and these paths), this current contribution needs to be improved and extended to avoid any empty path between the control transitions and these time-critical paths. A second assumption on the parts of the TEG that was exposed to the temporal constraints is relaxed in the majority of the previous results like in (Atto et al., 2011), (Amari et al., 2012). Their researches had considered only the particular places exposed to constraints. The present study manages to treat the problem of time constraints on some paths of the Timed Event Graph, which are not necessarily empty.

Accordingly, this paper is organized as follows; the basic definitions and notations concerning the formalism of Min-Plus algebra, Timed Event Graphs models and their Min-Plus linear representations, which are recalled in section 2 . The state feedback control problem is formulated in section 3 and the proposed feedback approaches are developed in section 4 . In particular, after considering the case of TEG with one input transition and a single temporal constraint on the path, the method is extended to the case where different constraints and several control transitions can be found. Section 5 is devoted to an illustrative application and finally section 6 is dedicated to conclude the contribution and to expose some future perspectives.

\section{Basic Definitions}

The paradigm of Petri nets is known to be a powerful tool used for the modeling and the analysis of discrete event systems. They are particularly used to represent phenomena such as synchronization and concurrency.

According to the context of analysis, many classes of Petri nets with more or less elaborate semantics are used. Comparatively, a basic class of Petri nets called Timed Event Graphs is the most studied. Its major benefit over many other timed Petri models is that their early behavior can be represented by linear equations in dioid algebra; Max- Plus and Min- plus (Baccelli et al., 1992). This leads to a large theory for linear systems where many concepts and theories have been involved the Timed Event Graphs.

\subsection{Formalism of Min-Plus Algebra}

In order to describe the state evolution of Discrete Event Systems, Min-Plus algebra can be seen as an adequate algebraic setting that involves synchronization, delays and parallelism phenomena. They are often observed in manufacturing assembly lines, transportation systems and communications networks. This tool is a mathematical formalism that paves the way for linear modeling. If these systems were described by differential or different equation in conventional algebra, they would have a nonlinear 
representation. This subsection provides the basic definitions and notations of Min-Plus algebra explored in this work.

Both minimization and usual addition are considered as elementary operations of the Min-Plus formalism. The Min-Plus algebra (or dioid Min-Plus), denoted $\overline{\mathbb{R}}_{\text {min }}$ is a commutative dioid for which the law $\oplus$ is the operation Min, having the neutral element $\varepsilon=+\infty$, and the second law $\oplus$ is the usual addition, with the neutral element $e=0$.

If $n \in \overline{\mathbb{R}}$ and $v, w \in \overline{\mathbb{R}}_{\text {min }}^{n}$, one denotes by $v \oplus w$, the vector with components $v_{i} \oplus w_{i}=\min \left(v_{i}, w_{i}\right)$ for $i=1$ to $n$. Taking $p, q \in \mathbb{N}$, and the matrices $A \in \overline{\mathbb{R}}_{\min }^{p \times n} \quad B \in \overline{\mathbb{R}}_{\text {min }}^{n \times q}$ the notations $A . B$ or just $A B$ denote both the matrix multiplication in $\overline{\mathbb{R}}_{\text {min }}$, which is defined by the following equation:

$$
(A \otimes B)_{i j}=(A . B)_{i j}=\bigoplus_{k=1}^{n}\left(A_{i k} \otimes B_{k j}\right)=\min _{k}\left(A_{i k}+B_{k j}\right) .
$$

The Kleene star of a square matrix $A \in \overline{\mathbb{R}}_{\text {min }}^{n \times n}$, denoted $A^{*}$, is defined by the following expression:

$$
A^{*}=\bigoplus_{i \in \mathbb{N}}\left(A^{i}\right),
$$

where $A^{0}$ denotes the unit matrix, where entries are equal to $e$ on the diagonal, and $\varepsilon$ elsewhere. Let us recall that $\nu \in \overline{\mathbb{R}}_{\text {min }}^{n}$ then $x=A^{*} . v$ is the maximal solution of both the inequality, $x \leq A . x \oplus v$, and also the equality, $x=A \cdot x \oplus v$.

The reader is invited to check out the book of Murata (1989), for an exhaustive presentation and further details on the theory of Min-Plus algebra.

\subsection{Modeling of Timed Event Graphs}

In (Murata, 1989) the Event Graphs are defined as a class of Petri nets where each place has exactly one upstream transition and one downstream transition. If time delays are combined with the places, then P-Timed Petri nets are found particularly within the class of Timed Event Graphs.

A Petri net is assessed to be live for an initial marking if all transitions can always be enabled by a future marking, as it is stated in (Baccelli et al., 1992).
The marking defines the dynamics of the evolution of the states inside a Timed Event Graph.

An active state is analogous to a token possession by the corresponding place.

An autonomous event graph is an event graph with only internal transitions.

According to Murata (1989), an autonomous event graph is called live whether in each of its circuits bears at least one token with respect to the initial marking.

An enabled transition comes to remove one token from its upstream place and to add it to each downstream place.

An empty path is a sequence of transitions and places consecutively connected by arcs, for which the cumulated marking is null.

In the overall, the following notations are used: while $P$ will denote the set of places of the considered graph, $T$ is its set of transitions. The number of transitions having at least one upstream place is denoted as $n$, whereas $m$ stands for the number of source transitions, having no upstream place. If $t_{i}, t_{j} \in T$, the place connecting $t_{j}$ to $t_{i}$ is denoted $p_{i j}$, if any, the corresponding delay is denoted $\tau_{i j}$ and the marking of this place is denoted $m_{i j}$. The maximal delay arising in the Timed Event Graph is denoted $\tau^{\max }$. In the present case, it is assumed that all the arcs of the TEG, must have weight 1 .

Let $S$ be a sequence of transitions and places having the following form: $\left(t_{i}, p_{i k_{1}}, t_{k_{1}}, p_{k_{1} k_{2}}, t_{k_{2}}, \ldots, t_{j}\right)$ where $p_{i k_{1}}, p_{k_{1} k_{2}}, \ldots \in P$ and $t_{i}, t_{k_{1}}, \ldots \in T$, as $\tau_{\alpha}$ is the sum of the existent delays along the path $\alpha$.

One could assume that $S$ is assimilated to a path denoted as $\alpha$.

Each transition of the considered Timed Event Graph is associated with a function of time that corresponds to the cumulated number of firings of the transition at a $t$ time.

The counters that correspond to source transitions within the TEG form the components of the vector $u(t)$, like $u(t) \in \overline{\mathbb{R}}_{\text {min }}^{m}$. Counters of other transitions form the components of the vector denoted as $\theta(t) \in \overline{\mathbb{R}}_{\text {min }}^{n}$, where $m$ represents the 
number of control transitions without any other upstream transition and $n$ stands for the number of transitions owning at least one upstream place (see Baccelli et al., 1992).

The dynamical behavior of a Timed Event Graph can be expressed by means of a linear equation over $\overline{\mathbb{R}}_{\text {min }}$, as in the following equation (1):

$\theta(t)=\overbrace{\tau=0}^{\tau^{\max }}\left(A_{\tau} \cdot \theta(t-\tau) \oplus B_{\tau} \cdot u(t-\tau)\right)$

$A_{\tau} \in \overline{\mathbb{R}}_{\text {min }}^{n \times n}$ is a matrix where entries $A_{\tau, i j}$ are equal to $m_{i j}$, the number of initial tokens in place $p_{i j}$, if this place exists and as the associated delay is $\tau$, or $\varepsilon$ else. Similarly, the entries of matrices $B_{\tau} \in \overline{\mathbb{R}}_{\min }^{n \times m}$ correspond to the initial tokens' number of the places directly followed by the control transitions.

As equation (1) is implicit in general, it is worthy to replace it with the following explicit equation:

$\theta(t)=\underset{\tau>0}{\oplus}\left(A_{0}^{*} \cdot A_{\tau} \cdot \theta(t-\tau) \oplus A_{0}^{*} \cdot B_{\tau} \cdot u(t-\tau)\right)$,

where $A_{0}^{*}$ is the Kleene star of $A_{0}$, as it has already been defined in the previous subsection.

\subsection{Min-Plus State Representation of TEGs}

Similar to the case of usual linear systems, the explicit equation (2) can be brought into the state space form, if all the delays in the Timed Event Graph are commensurable to a single delay. Consequently, one can assume without loss of generality that this elementary delay is equal to 1 .

In order to get a state space model from the TEG model, all the places with delays $\tau>1$ are firstly expanded into a number of $\tau$ places with delays equal to 1 .Therefore, $(\tau-1)$ intermediate transitions are added. The holding times in places are assumed integers. $x(t)$ denotes the resulting extended state vector. The dynamic behavior of the expanded Timed Event Graph is described by the equation:

$x(t)=\hat{A}_{0} \cdot x(t) \oplus \hat{A}_{1} \cdot x(t-1) \oplus \hat{B} \cdot u(t)$,

It can be rewritten into the following explicit equation:

$x(t)=A \cdot x(t-1) \oplus B \cdot u(t)$,

where $A$ and $B$ matrices expressions are given as $A=\hat{A}_{0}^{*} \cdot \hat{A}_{1}$ and $B=\hat{A}_{0}^{*} . \hat{B}$. Depending on the input $u(t)$ and on some initial conditions, all these notations permit to determine the deterministic behavior of the controlled Timed Event Graph. As this dependence can be explicit, the following formulation shall be used:

$x(t)=A^{\tau} \cdot x(t-\tau) \oplus\left[\bigoplus_{k=0}^{\tau-1} A^{k} \cdot B \cdot u(t-k)\right]$,

holding true, for every $\tau \geq 1$.

In the following, one assumes that the input $u(t)$ is actually a control, which can be arbitrarily assigned. For instance in a production process, the input can correspond to the authorization of performing a certain operation, primary materials input detection. Typically, the beginning of a task performed by a robot could be subject to such a control input for instance.

\section{Example 1}

Below, the illustrative example reinforces the already elaborated definitions.

Figure 1 illustrates the Timed Event Graph.

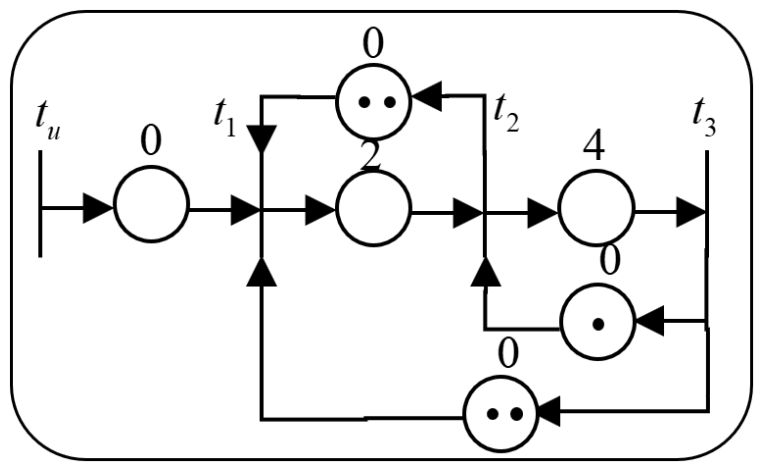

Figure 1. A Timed Event Graph example

Assuming that transitions' firing is performed with maximal possible speed, equation (1), in this graph leads to:

$\theta(t)=A_{0} \cdot \theta(t) \oplus A_{1} \cdot \theta(t-1) \oplus A_{2} \cdot \theta(t-2) \oplus A_{4} \cdot \theta(t-4)$

$\oplus B . u(t)$,

with:

$$
\begin{aligned}
A_{0} & =\left(\begin{array}{lll}
\varepsilon & 2 & 2 \\
\varepsilon & \varepsilon & \varepsilon \\
\varepsilon & \varepsilon & \varepsilon
\end{array}\right), A_{1}=\left(\begin{array}{lll}
\varepsilon & \varepsilon & \varepsilon \\
\varepsilon & \varepsilon & \varepsilon \\
\varepsilon & \varepsilon & \varepsilon
\end{array}\right), \\
A_{2} & =\left(\begin{array}{lll}
\varepsilon & \varepsilon & \varepsilon \\
e & \varepsilon & \varepsilon \\
\varepsilon & \varepsilon & \varepsilon
\end{array}\right), A_{4}=\left(\begin{array}{lll}
\varepsilon & \varepsilon & \varepsilon \\
\varepsilon & \varepsilon & \varepsilon \\
\varepsilon & e & \varepsilon
\end{array}\right), B=\left(\begin{array}{l}
e \\
\varepsilon \\
\varepsilon
\end{array}\right)
\end{aligned}
$$


The explicit equation (2) hence leads to:

$$
\begin{aligned}
& \theta(t)=\left(\begin{array}{lll}
\varepsilon & \varepsilon & \varepsilon \\
\varepsilon & \varepsilon & \varepsilon \\
\varepsilon & \varepsilon & \varepsilon
\end{array}\right) \theta(t-1) \oplus\left(\begin{array}{lll}
2 & \varepsilon & \varepsilon \\
e & \varepsilon & \varepsilon \\
\varepsilon & \varepsilon & \varepsilon
\end{array}\right) \theta(t-2) \oplus \\
& \left(\begin{array}{lll}
\varepsilon & 2 & \varepsilon \\
\varepsilon & \varepsilon & \varepsilon \\
\varepsilon & e & \varepsilon
\end{array}\right) \theta(t-4) \oplus\left(\begin{array}{l}
e \\
\varepsilon \\
\varepsilon
\end{array}\right) . u(t)
\end{aligned}
$$

After extending the initial graph to maintain a graph with delays normalized to 0 or 1 , the graph of Figure 2 is achieved.

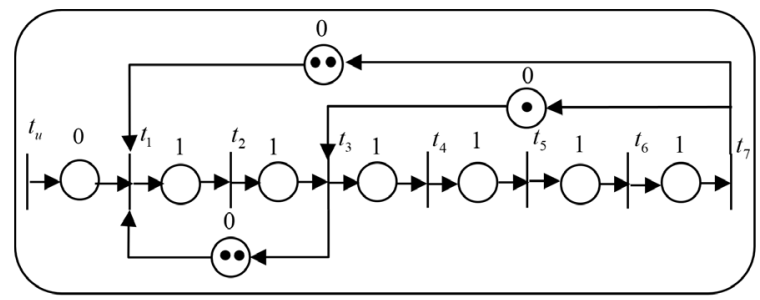

Figure 2. The extended equivalent TEG

Accordingly, the resulting state space equation is given as following:

$$
x(t)=\left[\begin{array}{lllllll}
\varepsilon & \varepsilon & \varepsilon & 2 & \varepsilon & \varepsilon & 2 \\
\varepsilon & \varepsilon & \varepsilon & e & \varepsilon & \varepsilon & 1 \\
\varepsilon & \varepsilon & \varepsilon & \varepsilon & \varepsilon & \varepsilon & e \\
e & \varepsilon & \varepsilon & \varepsilon & \varepsilon & \varepsilon & \varepsilon \\
\varepsilon & e & \varepsilon & \varepsilon & \varepsilon & \varepsilon & \varepsilon \\
\varepsilon & \varepsilon & \varepsilon & \varepsilon & e & \varepsilon & \varepsilon \\
\varepsilon & \varepsilon & \varepsilon & \varepsilon & \varepsilon & e & \varepsilon
\end{array}\right] x(t-1) \oplus\left[\begin{array}{c}
e \\
\varepsilon \\
\varepsilon \\
\varepsilon \\
\varepsilon \\
\varepsilon \\
\varepsilon
\end{array}\right] u(t)
$$

Linear systems over Min-Plus algebra could have been also used for performance evaluation like in (Gaubert, 1997), diagnosis, scheduling as the case of (van den Boom et al., 2013) to control various Discrete Event Systems, whose evolution is interpreted by Timed Event Graphs, for instance in (Akian et al., 2003), (Hardouin et al., 2010) and (Heidergott et al., 2006).

\section{Problem Formulation}

\subsection{Constrained Paths}

The majority of the existent industrial systems are subject to strict time duration constraints, which could be considered locally to some of their paths. Additional conditions are provided to check the compliance with the critical process. This needs to be formulated in order to provide efficient control laws through the constrained path formulation using Min-Plus algebra.

This study addresses the control of TEG under time constraints. In fact, in addition to minimal holding times for tokens in places of the TEG, there may be upper bounds on the time that tokens are allowed to spend in places. Moreover, such time constraints may additionally be imposed on paths within the TEG.

In such, one considers $\beta_{i j}$ as the path subject to a strict constraint like $t_{j \beta}$ and $t_{i \beta}$ respectively, and the upstream and the downstream transition of this path. A time interval $\left[\tau_{i j \beta}^{\min }, \tau_{i j \beta}^{\max }\right]$ is associated to this path, where $\tau_{i j \beta}^{\min }=\tau_{i j \beta}$.

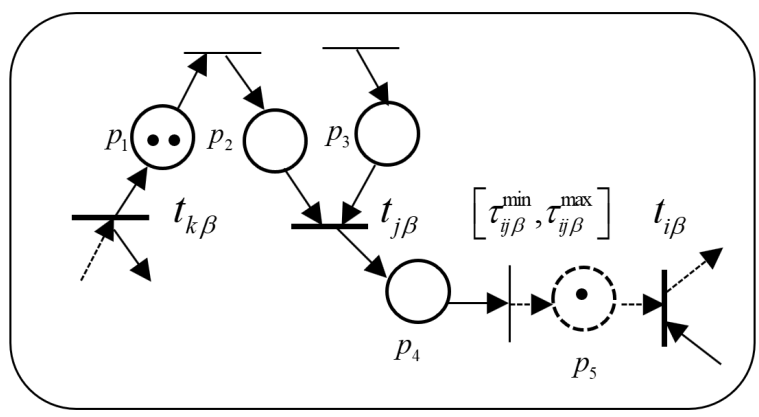

Figure 3. An example of a constrained Path

Through the path going from $t_{j \beta}$ to $t_{i \beta}$ illustrated by Figure 3, the temporal constraint is indeed expressed by the following inequality:

$x_{i \beta}(t) \geq m_{i j \beta} x_{j \beta}\left(t-\tau_{i j \beta}^{\max }\right)$

where $m_{i j \beta}$ is the sum of all the initial marking of places that are part of path $\beta_{i j}$. $\tau_{i j \beta}^{\max }$ defines the maximal upper bound, which is available over $\overline{\mathbb{R}}_{\text {min }}$ and represents the sum of all the delays associated with the places of the path. It is also the additional constraint that is required to be satisfied.

\subsection{Formulation of the Control Problem}

Strict time constraints are frequent in industrial processes. For instance, considering the example of a production process in a furnace, products should get specific thermal treatment, and the duration has to be fixed or even delimited by a time interval. Therefore, an adequate control that respects such critical constraints needs to be chosen. Figure 4 shows approximately the structure of a controlled constrained system. A feedback controller of the form: $u(t)=F . x(t-1)$ directly linked to the input is applied. 
The definition of a Timed Event Graph takes into consideration a delay on each place that corresponds to a minimal holding time. The maximal duration emerges as an additional time constraint that should be checked indeed. Accordingly, the question of verification is tackled as a control problem formulation.

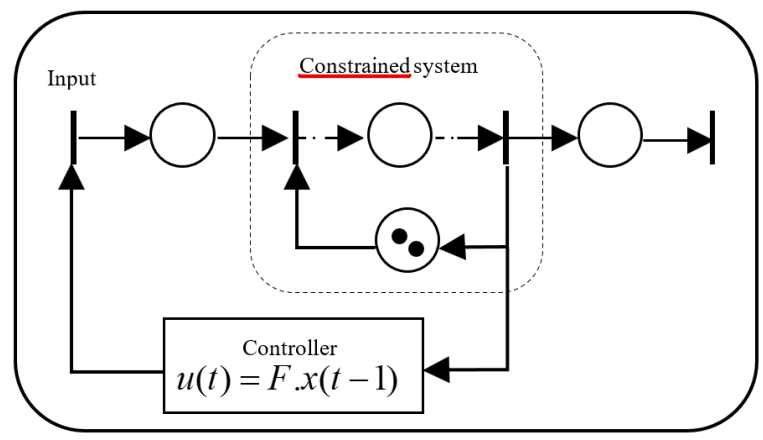

Figure 4. A structure of a controlled constrained system

Remark 1. A static control law like $u(t)=G \cdot x(t)$ could lead to implicit loops. Because the solution is characterized by infinite rather then single loops, this control problem lacks the adequate definition. On one hand, using $u(t)=G \cdot x(t)$, a badly posed feedback emerge. On the other hand a feedback of the form $u(t)=F \cdot x(t-1)$ is always well posed, which ensures the closed loop $u(t)=(A \oplus B \cdot F) \cdot x(t-1)$.

\section{State Feedback Control of TEG}

As the response time of most modern industrial systems is crucial, it is worthy to seek for a control that solves the problem of the temporal constraint. In doing so, all the parts of an operational system must be taken into account once they are under time constraint.

Within the framework of this paper, the main objective of the control synthesis is to confirm that the sojourn time remains dependent on some durations or time limits in certain paths of the TEG. Thereupon, these temporal constraints have not necessarily been respected without suitable control.

A new method for the synthesis of feedback control laws ensuring the respect of temporal constraints on paths using Min-Plus algebra is addressed by checking the sufficient conditions. The approach towards this method of control law synthesis is based on (Ben Afia et al., 2019). Accordingly, the present work consists of generalizing the Timed
Event Graphs subjected to several time constraints and multiple controls.

\subsection{TEG with One Control Transition and a Single Constrained Path}

In order to find a suitable feedback respecting the critical process, adequate control laws should be sought. Therefore, within this context, an entire sub-process or a task within an industrial process could be exposed to strict time duration. Some previous approaches related to the synthesis of feedback control laws for discrete event systems, have two limitative assumptions as in (Tebani et al., 2017) and (Amari, 2015). The first one considers an empty cumulated marking through the path between the resource transition and the upstream transition of the time critical path. The second one supposes that the constrained place is unmarked. Otherwise, in the present case, these works are extended and these assumptions are relaxed by keeping meanwhile the basic properties of the Timed Event Graphs such as liveness.

At the beginning, the case of one control transition and a single constrained path is tackled. Then, it is expanded to a general case including multiple constrained paths and also multiple controls.

For that, one defines:

$\alpha$ as the path delimiting the input transition of the TEG and the upstream transition of the constraint path $\beta$.

$\tau_{\alpha}$ and $m_{\alpha}$ are the non-zero cumulated delay and the sum of the markings along the path $\alpha$, respectively.

$u(t)$ and $x_{j \beta}(t)$ denote the counter functions of $t_{u}$ and $t_{j \beta}$, respectively.

The counter functions' relationship between $u(t)$ and $x_{j \beta}(t)$ is expressed by the following inequality:

$x_{j \beta}(t) \leq m_{\alpha} u\left(t-\tau_{\alpha}\right)$

Considering $\tau=\tau_{\alpha}$ and from the definition of $t_{u}$ and $t_{j \beta}$, one has:

$x_{j \beta}(t) \leq\left(A^{\tau_{\alpha}} B\right)_{u j \beta} x_{u}\left(t-\tau_{\alpha}\right)$.

As $x_{u}(t) \leq u(t)$ from which it is quite evident that:

$x_{j \beta}(t) \leq\left(A^{\tau_{\alpha}} B\right)_{u j \beta} u\left(t-\tau_{\alpha}\right)$ 
Reapplying again (4) with $\tau=\phi$ the following explicit expression is obtained:

$x_{i \beta}(t)=\bigoplus_{r=1}^{N}\left(A^{\phi}\right)_{i \beta r} x_{r}(t-\phi) \oplus\left[\bigoplus_{k=1}^{\phi-1}\left(A^{k} B\right)_{i \beta} u(t-k)\right](7)$

Available for every integer $\phi \geq 1$, as $r=1$ to $N$, this represents the key to obtain the following result.

Theorem 1. Assuming that a Timed Event Graph, whose evolution is modeled by the MinPlus linear equation (3), it is subject to a single temporal constraint of the form (5) imposed on the path $\beta_{i j}$.

By taking $\phi=\tau_{i j \beta}^{\max }+\tau_{\alpha}+1$ from the following control laws:

$u(t) \leq \bigoplus_{r=1}^{N}\left(\left(A^{\phi}\right)_{i \beta r}-\left(A^{\tau_{\alpha}} B\right)_{u j \beta}-m_{i j \beta}\right) \cdot x_{r}(t-1)$,

One could define the causal control laws that ensure the respect of the constraint (5), only if this condition holds true:

$\left(A^{k} B\right)_{i \beta} \geq\left(A^{\tau_{\alpha}} B\right)_{u j \beta}+m_{i j \beta}$, for each $k=0$ to $(\phi-1)$

Proof. Taking into account equation (7), it's evident that path's constraint (5) is respected only when the follwing condition holds:

$$
\underset{k=1}{\oplus-1}\left(A^{k} B\right)_{i \beta} u\left(t-k+\tau_{i j \beta}^{\max }\right) \geq m_{i j \beta} x_{j \beta}(t)
$$

Moreover, if equation (6) is considered, the previous condition will become as following:

$\underset{k=1}{\oplus-1}\left(\left(A^{k} B\right)_{i \beta}-m_{i j \beta}\right) u\left(t-k+\tau_{i j \beta}^{\max }\right) \geq\left(A^{\tau_{\alpha}} B\right)_{u j \beta} u\left(t-\tau_{\alpha}\right)$

It represents an enough condition depending exclusively on the previous path $\alpha$, as well as on the initial markings of the path $\beta_{i j}$.

\section{Remark 2.}

Considering $F_{r}=\left(A^{\phi}\right)_{i \beta r}-\left(A^{\tau_{\alpha}} B\right)_{u j \beta}-m_{i j \beta}$, the suitable feedback $F$ of the controller $u(t)=F x(t-1)$, is found by choosing $F_{r}=\varepsilon$ for $\left(A^{\phi}\right)_{i \beta r}=\varepsilon$ and $F_{r}=\max \left(e,\left(A^{\phi}\right)_{i \beta r}-\left(A^{\tau_{\alpha}} B\right)_{u j \beta}-m_{i j \beta}\right)$ for $\left(A^{\phi}\right)_{i \beta r} \neq \varepsilon$, where $F_{r}$ represents the component of the vector $F$.

\subsection{Control Synthesis for the Case of Multiple Constrained Paths}

This subsection deals with the control synthesis of a Timed Event Graph, while having one source transition, such that there exist $\mathrm{Z}$ constrained paths, denoted as $\beta_{z}$, for $\mathrm{z}=1$ to $\mathrm{Z}$, respectively. For each constrained path let $m_{\beta z}, \tau_{\beta z}$ and $\tau_{\beta_{2}}^{\max }$, denote the initial marking, the minimal and maximal delays of the constrained path, respectively. Further, let $t_{\beta z}$ and $t_{\beta z}^{\prime}$ denote the input and output transitions of the path $z$, respectively. $x_{z}$ and $x_{z}^{\prime}$ denote the corresponding transitions' counter functions, and $\lambda_{\beta z}$ indicates the cumulated delay along a path going from $t_{u}$ to $t_{\beta z}$. These constraints are expressed by the inequalities given as following:

$x_{\beta_{z}}^{\prime}(t) \geq m_{\beta_{z}} x_{\beta_{z}}\left(t-\tau_{\beta_{z}}^{\max }\right)$

for $z=1$ to $Z$.

$u_{z}(t)$ is denoted as the control law that satisfies all the corresponding constraints on the paths, given by the inequalities (8).

Theorem 2. As a Timed Event Graph modeled by Min-Plus linear equation (3) and submitted to temporal constraints imposed on $\mathrm{Z}$ paths is considered, the causal controls ensuring the respect of all constraints are computed as follow:

$u(t) \leq \bigoplus_{z=1}^{Z} u_{z}(t)$

$u_{z}(t)=\bigoplus_{r=1}^{N}\left[\left(\left(A^{\phi_{\beta z}}\right)_{x^{\prime} u}-\left(A^{\lambda_{\beta z}} B\right)_{x u}-m_{\beta_{z}}\right) x_{r}(t-1)\right]$, is

the control law ensuring the respect of the $Z$ constraints on the paths $\beta$, knowing that:

$\left(\phi_{\beta_{z}}=\tau_{\beta_{z}}^{\max }+\lambda_{\beta_{z}}+1\right)$,

only if the below conditions are satisfied:

$$
\left(A^{k} B\right)_{x^{\prime} u} \geq\left(A^{\lambda_{\beta_{z}}} B\right)_{x u}+m_{\beta_{z}} \text { for } k=1 \text { to }\left(\phi_{\beta_{z}}-1\right)
$$

Proof. By admitting that the control law provided by $u(t)$ ensures the $z^{\text {th }}$ constraint on the path,with Theorem 1, the global control law will be the following:

$u(t) \leq \underset{z=1}{Z} u_{z}(t)$,

Every $z=1$ to $Z$, will lead quite natural to the satisfaction of simultaneous constraints if the provided conditions are satisfied. 


\subsection{Generalization for the Case of Multiple Controls}

This subsection deals with a Timed Event Graph with $m$ existing source transitions. Firstly, it is supposed that $\beta_{i j}$ is the single path subjected to the additional upper bound of the path's temporal constraint.

In this context, a vector, $u(t) \in \overline{\mathbb{R}}_{\text {min }}^{m}$ with $m \geq l$ is calculated which is a control law that must satisfy the constraint (5). To each source transition, a variable counter is associated, denoted by $u_{s}(t)$, for each $s \in[1, m] . \lambda_{s}$ notes the cumulated delay along each path going from $t_{u_{\mathrm{s}}}$ to $t_{j \beta}$, which denote the source transitions and each upstream transition of the path $\beta_{i j}$, respectively.

Theorem 3. Regarding the Min-Plus system (3) that describes the behavior of a TEG subject to a single constraint of the form (5) on one path, the below inequality can be obtained:

$u_{s}(t) \leq \bigoplus_{r=1}^{N}\left[\left(\left(A^{\phi_{s}}\right)_{i r}-\left(A^{\lambda_{s}} B\right)_{j \beta} u_{s}-m_{i j \beta}\right) x_{r}(t-1)\right]$

With $\left(\phi_{s}=\tau_{i j \beta}^{\max }+\lambda_{s}+1\right)$, the feedback controls ensuring the respect of the temporal constraint can be defined the following condition is checked:

$\left(A^{k} B\right)_{i \beta r^{\prime}} \geq\left(A^{\lambda_{s}} B\right)_{j \beta u_{i}}+m_{i j \beta}$,

with $k \in\left[1,\left(\phi_{s}-1\right)\right]$ and $i^{\prime} \in[1, m]$

Proof. By replacing $\tau$ by $\lambda_{s}$ in (4), and from the definition of the counter function of $t_{j \beta}$, it is obtained:

$x_{j \beta}(t) \leq\left(A^{\lambda_{s}} B\right)_{j \beta u_{z}} u_{s}\left(t-\lambda_{s}\right)$

Following the same equation (4), by substituting $\tau:=\phi_{s}$, one gets:

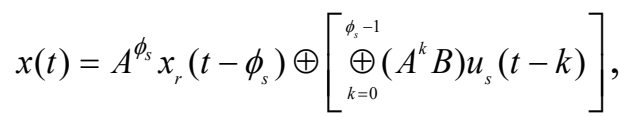

in particular the following explicit equation can be deduced:

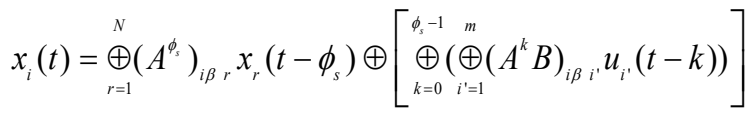

Considering equation (10), particularly, the second part, the temporal constraint given by (5), is satisfied only if the following condition holds true:

$$
\underset{k=0}{\phi_{s}-1}\left(\left(_{i^{\prime}=1}^{m}\left(A^{k} B\right)_{i \beta i^{\prime}} u_{i^{\prime}}\left(t-k+\tau_{i j \beta}^{\max }\right)\right) \geq m_{i j \beta} x_{j \beta}(t) .\right.
$$

Further, taking (9) into account, this condition becomes:

$$
\begin{aligned}
& \left.\bigoplus_{k=0}^{\phi_{s}-1}\left[\bigoplus_{i=1}^{m}\left(\left(A^{k} B\right)_{i \beta i^{\prime}}-m_{i j \beta}\right) u_{i^{\prime}}\left(t-k+\tau_{i j \beta}^{\max }\right)\right)\right] \\
& \geq\left(A^{\lambda} B\right)_{j \beta u_{s}} u_{s}\left(t-\lambda_{s}\right)
\end{aligned}
$$

By choosing $\left(\phi_{s}=\tau_{i j \beta}^{\max }+\lambda_{s}+1\right)$, the previous given condition is verified, and the control law satisfies the inequality (5), thus, it can be checked if that the temporal constraint on the path is also respected.

Theorem 4. Assuming that the Timed Event Graph is characterized by $m$ source transitions as $m>1$ and disposed to $z$ additional temporal constraints on some of its paths of the form (8), the causal control law guaranteeing the respect of all the $\mathrm{Z}$ constraints will be defined as follows:

$u_{l}(\mathrm{t}) \leq \bigoplus_{z=1}^{Z} u_{z}(t)$.

Thus, $\mathrm{z}=1$ to $\mathrm{Z}$ where $u_{z}(t)$ is the control law, already calculated by theorem 2 , to check the $z^{\text {th }}$ constraint and $u_{l}(t)=\varepsilon$ for $l \neq z$.

$u_{l}(t)=\bigoplus_{z=1}^{Z} u_{z}(t)$

Proof. A control law $u_{z}(t)$ validates the $z^{\text {th }}$ constraint, if the condition given by theorem 2 is satisfied. Accordingly, one obtains:

$u_{z}(t)=\bigoplus_{z=1}^{Z} u_{z}(t)$

for $Z=1$ to $Z$.

According to Theorem 4, it is clear that the control law:

$u_{l}(\mathrm{t}) \leq \bigoplus_{z=1}^{Z} u_{z}(t)$

available for $l=z$ to $Z$, guarantees the respect of all $\mathrm{Z}$ existing temporal constraints of the TEG. 


\section{Application to a Manufacturing Cell}

\subsection{Modeling and Control of the Manufacturing Cell}

In this subsection, the above theoretical results are applied to the case of a manufacturing cell inside a flexible workshop. Therefore, Figure 5 provides the simplified structure of the basic parts of the application.

The automated cell consists of 7 areas $\left(\mathrm{C}_{10}, \mathrm{C}_{20}\right.$, $\left.\mathrm{C}_{1}, \mathrm{C}_{2}, \mathrm{C}_{3}, \mathrm{C}_{4}, \mathrm{C}_{5}\right)$ : two input areas $\left(\mathrm{C}_{10}\right.$ and $\left.\mathrm{C}_{20}\right)$, two supplying and operating areas, represented by $\left(\mathrm{C}_{1}\right.$ and $\left.\mathrm{C}_{2}\right)$, respectively. As each area contains one single processing machine, each one serves to occupy a particular task.

Both of the conveyors $\mathrm{C}_{3}$ and $\mathrm{C}_{4}$ serve to transfer pieces, which have already been treated. Therefore, each piece gets a specific operation within two different processes 1 and 2, whereas $\mathrm{C}_{5}$ illustrates a convergence area, devoted for pieces evacuation.

It should be specified that conveyors $\mathrm{C}_{1}, \mathrm{C}_{2}, \mathrm{C}_{3}$ and $\mathrm{C}_{4}$ are always running, since they are characterized by their capacities, denoted as $k_{1}, k_{2}, k_{3}$, and $k_{4}$, which are represented through places $p_{3} p_{13} p_{17}$ and $p_{7}$, respectively.

- Actuators $\mathrm{A}_{1}-\mathrm{A}_{5}$ ensure loads regulation.

- The whole manufacturing cell is controlled by a centralized Programmable Logic Controller PLC, which uses output data from sensors to ensure a specific operating cycle by a set of Actuators.

- By means of specific proximity sensors $S_{1}$ and $\mathrm{S}_{4}$. Products entry on the conveyors lines $\mathrm{C}_{10}$ and $\mathrm{C}_{20}$ is detected.

- After carrying products from a buffer as well as after being blocked when $\mathrm{C}_{10}$ and $\mathrm{C}_{20}$ reach their limit capacities, they are transferred by $\mathrm{C}_{1}$ and $\mathrm{C}_{2}$ to $\mathrm{M}_{1}$ and $\mathrm{M}_{2}$. It is noticed that the two machines perform different processes with different speed rates.

- When actuators $\mathrm{A}_{1}$ and $\mathrm{A}_{4}$ are abled, a read write system is activated to read the label of the product and process its specific production plan.

- $\mathrm{S}_{2}$ is multiplexed photoelectric proximity sensor with two transmitters and one receiver. Its mere function is the detection of the entrance of products that have already been delivered from machines $\mathrm{M}_{1}$ or $\mathrm{M}_{2}$.

- Products are then loaded on $C_{3}$ and $C_{4}$, two conveyors with different load capacities

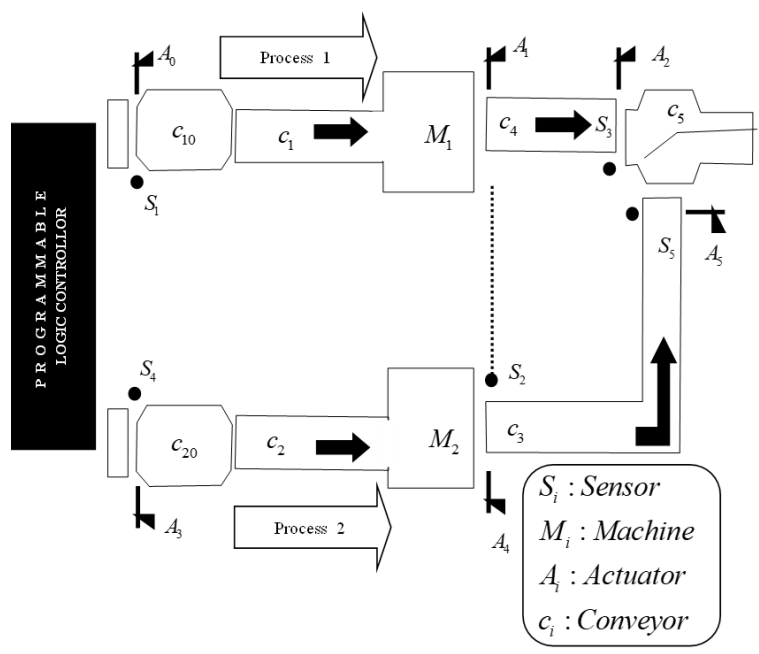

Figure 5. A simplified representation of the manufacturing cell

Figure 6 exposes the graphical modeling of the application by means of the Timed Event Graph.

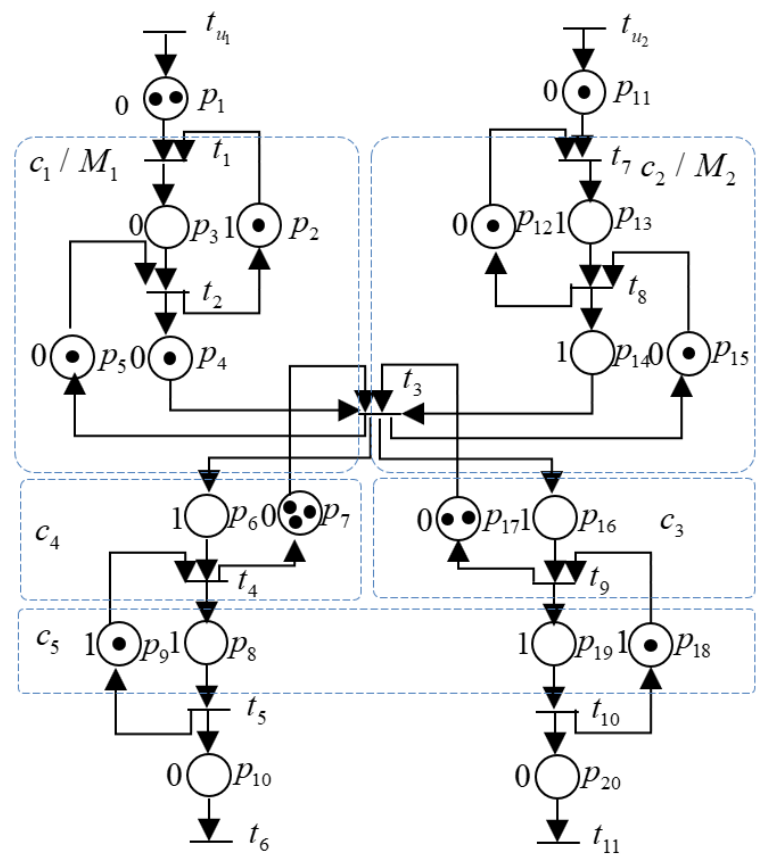

Figure 6. The TEG model of the manufacturing cell 
- $\quad t_{1}, t_{7}, t_{3}$ and $\left(t_{4}, t_{9}\right)$ are transitions corresponding to the entrance detection meaning of the sensors: $S_{1}, S_{4}, S_{2}, S_{3}$ and $S_{5}$ of individually $C_{1}$, $C_{2}, C_{3}, C_{4}$ and $C_{5}$.

- If sensors detect any failure, the PLC must immediately apply specific control with a set of actuators $\left(A_{2}, A_{5}\right)$, in order to avoid products collision on the common area $C_{5}$.

- Each product consists of different stock areas; $p_{10}$ and $p_{20}$ (not illustrated by Figure 5 for simplicity sake). Since the TEG in Figure 6 shows the existence of two source transitions, which are $\left(t_{u 1}, t_{u 2}\right)$, the system $m=2$ is modeled.

The manufacturing cell is subject to critical time duration. Considering that these constraints are applied to some of its path, they are denoted by $Z_{\beta 1}$ and $Z_{\beta 2}$, respectively. The first constrained path goes from $t_{1}$ to $t_{3}$ and the second one is between $t_{7}$ and $t_{3}$.

Regarding the maximum speed firing for each transition $t_{i}$, a counter function is associated, which constitutes the component of the vector $x(t)$.

Thereupon, the TEG meaning of Min-Plus system equations is modeled as follows:

$$
\left\{\begin{array}{l}
\theta_{1}(t)=2 \cdot u_{1}(t) \oplus 1 . \theta_{2}(t) \\
\theta_{2}(t)=e \cdot \theta_{1}(t-1) \oplus 1 . \theta_{3}(t) \\
\theta_{3}(t)=e \cdot \theta_{2}(t) \oplus e \cdot \theta_{8}(t-1) \oplus 3 . \theta_{4}(t) \oplus 2 . \theta_{9}(t) \\
\theta_{4}(t)=e \cdot \theta_{3}(t-1) \oplus 1 . \theta_{5}(t-1) \\
\theta_{5}(t)=e \cdot \theta_{4}(t-1) \\
\theta_{6}(t)=e \cdot \theta_{5}(t) \\
\theta_{7}(t)=1 . u_{2}(t) \oplus 1 . \theta_{8}(t) \\
\theta_{8}(t)=e \cdot \theta_{7}(t-1) \oplus 1 . \theta_{3}(t) \\
\theta_{9}(t)=e \cdot \theta_{3}(t-1) \oplus 1 . \theta_{10}(t-1) \\
\theta_{10}(t)=e \cdot \theta_{9}(t-1) \\
\theta_{11}(t)=e \cdot \theta_{10}(t)
\end{array}\right.
$$

Since the modeled system is $m>1$, the control law should be revealed in the form below:

$u(t)=\left(\begin{array}{l}u_{1}(t) \\ u_{2}(t)\end{array}\right)$

and must satisfy both constraints on paths.

Referring to the subsection 2.1, the state space representation is computed and it is illustrated by the following matrix system:

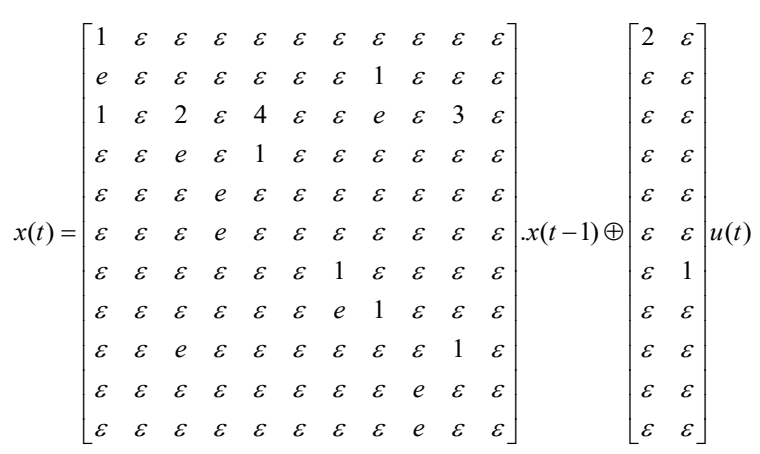

Beyond proceeding each constrained path, it is noticed that there is a component of the vector $u(t)$ that ensures the constraint.

For the constraint $Z_{\beta 1}$, the following inequality is given:

$x_{3 \beta_{1}}(t) \geq 1 . x_{1 \beta_{1}}(t-1)$,

that represents the conveyor $c_{1}$ and the machine $M_{1}$, where its path is delimited by transitions starting from $t_{1}$ to $t_{3}$. The upper limit that should be respected is defined as:

$\tau_{i j \beta}^{\max }=\tau_{31 \beta}^{\max }=1$,

As $m_{i j \beta_{1}}$ is the cumulated marking through the constrained path, it is noticed that one token does exist on the place $P_{4}$. Thus, $m_{31 \beta_{1}}=1$ is obtained.

The second constraint $Z_{\beta 2}$ is represented by the following inequality: $x(t)_{3 \beta_{2}} \geq x_{7 \beta_{2}}(t-2)$.

On the other hand $m_{37 \beta_{2}}=e$ represents the cumulated empty marking and $\tau_{37 \beta_{2}}^{\max }=1$ is the second upper bound that must not exceed 1 time unit, on the other hand.

Since the existence of the two source transitions, precisely $t_{u_{1}}$ and $t_{u_{2}}$ from the TEG of the application, could be recognized in Figure 6, one obtains $m=2$.

The cumulated delay along the path bounded by $t_{u 1}$ and $t_{1}$ is denoted $\lambda_{1}=0 . m_{\alpha z_{1}}=2$ is the marking through it. Consequently, it can be chosen:

$\phi_{z_{1}}=\tau_{31 \beta}^{\max }+\lambda_{1}+1=2$

A second cumulated delay along the path bounded by tu 2 and $\mathrm{t} 7$ is determined which is also equal to $\lambda_{2}=0$, and $m_{\alpha z_{2}}=1$ is its marking. Accordingly, it can be chosen:

$\phi_{z_{2}}=\tau_{73 \beta}^{\max }+\lambda_{2}+1=3$ 
At present, the formulation that points out the deterministic behavior of the controlled Timed Event Graph should be determined. As in equation (4), it is presented as follows:

$$
x(t)=\left[\begin{array}{lllllllllll}
3 & \varepsilon & \varepsilon & \varepsilon & \varepsilon & \varepsilon & \varepsilon & \varepsilon & \varepsilon & \varepsilon & \varepsilon \\
2 & \varepsilon & \varepsilon & \varepsilon & \varepsilon & \varepsilon & 2 & 3 & \varepsilon & \varepsilon & \varepsilon \\
3 & \varepsilon & 3 & 6 & 5 & \varepsilon & 1 & 2 & 5 & 4 & \varepsilon \\
2 & \varepsilon & 1 & 4 & 2 & \varepsilon & e & 1 & 3 & 5 & \varepsilon \\
1 & \varepsilon & 2 & 1 & 4 & \varepsilon & \varepsilon & e & \varepsilon & 3 & \varepsilon \\
1 & \varepsilon & 2 & 1 & 4 & \varepsilon & \varepsilon & e & \varepsilon & 3 & \varepsilon \\
\varepsilon & \varepsilon & \varepsilon & \varepsilon & \varepsilon & \varepsilon & 3 & \varepsilon & \varepsilon & \varepsilon & \varepsilon \\
\varepsilon & \varepsilon & \varepsilon & \varepsilon & \varepsilon & \varepsilon & 2 & 3 & \varepsilon & \varepsilon & \varepsilon \\
2 & 1 & 4 & 6 & \varepsilon & e & 1 & 3 & 2 & \varepsilon & \varepsilon \\
1 & \varepsilon & 2 & 4 & \varepsilon & \varepsilon & \varepsilon & e & 1 & 3 & \varepsilon \\
1 & \varepsilon & 2 & 4 & \varepsilon & \varepsilon & \varepsilon & e & 1 & 3 & \varepsilon
\end{array}\right] x(t-3)
$$

Taking into consideration $\left(\tau=\phi_{\tau_{1}}=2\right)$, the explicit equation (4) for the first constrained path is expressed as following:

$$
\begin{aligned}
& x(t)=A^{\phi_{z_{1}}} \cdot x\left(\mathrm{t}-\phi_{z_{1}}\right) \oplus\left[\bigoplus_{k=0}^{\left(\phi_{z_{1}}-1\right)} A^{k} \cdot B \cdot u(t-k)\right] \\
& =A^{2} \cdot x(\mathrm{t}-2) \oplus\left[\bigoplus_{k=0}^{1} A^{k} \cdot B \cdot u(\mathrm{t}-k)\right] .
\end{aligned}
$$

Such that, one obtains:

$$
A^{2}=\left[\begin{array}{lllllllllll}
2 & \varepsilon & \varepsilon & \varepsilon & \varepsilon & \varepsilon & \varepsilon & \varepsilon & \varepsilon & \varepsilon & \varepsilon \\
1 & \varepsilon & \varepsilon & \varepsilon & \varepsilon & \varepsilon & \varepsilon & \varepsilon & \varepsilon & \varepsilon & \varepsilon \\
2 & \varepsilon & 4 & 4 & 6 & \varepsilon & e & 1 & 3 & 5 & \varepsilon \\
1 & \varepsilon & 2 & 1 & 4 & \varepsilon & \varepsilon & e & \varepsilon & 3 & \varepsilon \\
\varepsilon & \varepsilon & e & \varepsilon & 1 & \varepsilon & \varepsilon & \varepsilon & \varepsilon & \varepsilon & \varepsilon \\
\varepsilon & \varepsilon & e & \varepsilon & 1 & \varepsilon & \varepsilon & \varepsilon & \varepsilon & \varepsilon & \varepsilon \\
\varepsilon & \varepsilon & \varepsilon & \varepsilon & \varepsilon & \varepsilon & 2 & \varepsilon & \varepsilon & \varepsilon & \varepsilon \\
\varepsilon & \varepsilon & \varepsilon & \varepsilon & \varepsilon & \varepsilon & 1 & 2 & \varepsilon & \varepsilon & \varepsilon \\
1 & \varepsilon & 2 & \varepsilon & 4 & \varepsilon & \varepsilon & e & 1 & 3 & \varepsilon \\
\varepsilon & \varepsilon & e & \varepsilon & \varepsilon & \varepsilon & \varepsilon & \varepsilon & \varepsilon & 1 & \varepsilon \\
\varepsilon & \varepsilon & e & \varepsilon & \varepsilon & \varepsilon & \varepsilon & \varepsilon & \varepsilon & 1 & \varepsilon
\end{array}\right]
$$

Akin to the previous formulation, if we embrace the equation $\left(\tau=\phi_{z_{2}}=3\right)$, we can allow achieve:

$$
\begin{aligned}
& x(t)=A^{\phi_{z_{2}}} x\left(\mathrm{t}-\phi_{z_{1}}\right) \oplus\left[\bigoplus_{k=0}^{\left(\phi_{z_{2}}-1\right)} A^{k} \cdot B \cdot u(t-k)\right] \\
& =A^{3} \cdot x(\mathrm{t}-3) \oplus\left[\bigoplus_{k=0}^{2} A^{k} \cdot B \cdot u(\mathrm{t}-k)\right]
\end{aligned}
$$

After checking the sufficient conditions, according to Theorem 4, the first control law ensuring the respect of the constraint on the path is given as following:

$$
\left.u_{1}(t)=\bigoplus_{r=1}^{11}\left(\left(A^{2}\right)_{3 \beta_{1} r}-\left(A^{1}\right)_{u 1 \beta_{1}}-1\right) x_{r}(t-1)\right)
$$

The second control law $u_{2}(t)$ is also given as:

$$
\left.u_{2}(t)=\bigoplus_{r=1}^{11}\left(\left(A^{3}\right)_{3 \beta 2 r}-\left(A^{1}\right)_{u\urcorner \beta 2}\right) x_{r}(t-1)\right)
$$

While taking into account Remark 2, it is noticed that the control law prevents the violation of both constraints $Z_{\beta 1}$ and $Z_{\beta 2}$, commonly on the paths $\beta_{1}$ and $\beta_{2}$, and it is given by:

$$
u(t)=\left(\begin{array}{c}
e . x_{1}(t-1) \oplus 1 . x_{3}(t-1) \oplus 1 . x_{4}(t-1) \oplus 3 . x_{5}(t-1) \oplus \\
e . x_{7}(t-1) \oplus e . x_{8}(t-1) \oplus e . x_{9}(t-1) \oplus 2 . x_{10}(t-1) \\
2 x_{1}(t-1) \oplus 2 x_{3}(t-1) \oplus 5 x_{4}(t-1) \oplus 4 x_{5}(t-1) \oplus \\
e . x_{7}(t-1) \oplus 1 x_{8}(t-1) \oplus 4 x_{9}(t-1) \oplus 3 x_{10}(t-1)
\end{array}\right)
$$

for the first control law given by:

$u_{1}(t)=e . x_{1}(t-1) \oplus 1 . x_{3}(t-1) \oplus 1 . x_{4}(t-1) \oplus 3 . x_{5}(t-1)$

$\oplus e . x_{7}(t-1) \oplus e . x_{8}(t-1) \oplus e . x_{9}(t-1) \oplus 2 . x_{10}(t-1)$

which is also written as:

$u_{1}(\mathrm{t})=\operatorname{Min}\left(e . x_{1}(t-1) \oplus 1 . x_{3}(t-1) \oplus 1 . x_{4}(t-1) \oplus 3 . x_{5}(t-1) \oplus\right.$

e. $\left.x_{7}(t-1) \oplus e . x_{8}(t-1) \oplus e . x_{9}(t-1) \oplus 2 . x_{10}(t-1)\right)$

To simplify those control laws, the terms of each vector component are firstly compared. Obviously, by examining the components of the first vector, it is noticed that $x_{3}(t-1)$ and $x_{4}(t-1)$ share the same coefficients, and the firing time of $t_{3}$ exceeds that of $t_{4}$. The same analogy is applied to $t_{1} t_{7} t_{8}$ and $t_{9}$, where the firing times of $t_{1}$ and $t_{7}$ exceed that of $t_{8}$ and $t_{9}$.

The control law guaranteeing the first temporal constraint is simplified to the next one:

$u_{1}(t)=e . x_{1}(t-1) \oplus 1 . x_{3}(t-1) \oplus 3 \cdot x_{5}(t-1) \oplus e . x_{7}(t-1)$

$\oplus 2 . x_{10}(t-1)$. 
For the second component of the vector $u(t)$, the control law could be written by:

$u_{2}(t)=\operatorname{Min}\left(2 x_{1}(t-1) \oplus(\varepsilon-1) x_{2}(t-1) \oplus 2 x_{3}(t-1) \oplus 5 x_{4}(t-1)\right.$

$\oplus 4 x_{5}(t-1) \oplus(\varepsilon-1) x_{6}(t-1) \oplus e . x_{7}(t-1) \oplus 1 x_{8}(t-1) \oplus 4 x_{9}(t-1)$

$\left.\oplus 3 x_{10}(t-1) \oplus(\varepsilon-1) x_{11}(t-1)\right)$

It is assumed that this control law could be reduced as following:

$\operatorname{Min}\left(2 . x_{1}(t-1) \oplus 5 . x_{4}(t-1) \oplus e . x_{7}(t-1) \oplus 1 . x_{8}(t-1)\right.$

$\left.\oplus 4 . x_{9}(t-1) \oplus 3 . x_{10}(t-1)\right)$,

taking into account the following statements:

2. $x_{3}(t-1)$ and 2.x $x_{1}(t-1)$ share the same coefficient, and as $t_{1}$ firing time exceeds that of $t_{3}$ only the component of $t_{1}$ counter function is kept. The same thing applies for $t_{5}$ and $t_{9}$, since we heed that the firing time of $t_{9}$ is preceding that of $t_{5}$, there is a tendency to keep only the $t_{9}$ component. Consequently, the control law for $u_{2}(t)$ has been reduced to:

$u_{2}(t)=2 . x_{1}(t-1) \oplus 5 . x_{4}(t-1) \oplus e . x_{7}(t-1) \oplus 1 . x_{8}(t-1) \oplus 4 . x_{9}(t-1)$

$\oplus 3 . x_{10}(t-1)$.

Overall, simplifying the global control vector ensuring the respect of both constraints on paths including $\mathrm{C}_{1} \mathrm{M}_{1}$ process and $\mathrm{C}_{2} \mathrm{M}_{2}$ process, it is expressed by the below vector $u(t)=\left(\begin{array}{l}u_{1}(t) \\ u_{2}(t)\end{array}\right)$, it
is obtained:

$$
\begin{aligned}
& u_{1}(t)=e \cdot x_{1}(t-1) \oplus 1 . x_{3}(t-1) \oplus 3 . x_{5}(t-1) \oplus e . x_{7}(t-1) \\
& \oplus 2 . x_{10}(t-1) \\
& u_{2}(t)=2 \cdot x_{1}(t-1) \oplus 5 \cdot x_{4}(t-1) \oplus e . x_{7}(t-1) \oplus 1 . x_{8}(t-1) \\
& \oplus 4 . x_{9}(t-1) \oplus 3 \cdot x_{10}(t-1)
\end{aligned}
$$

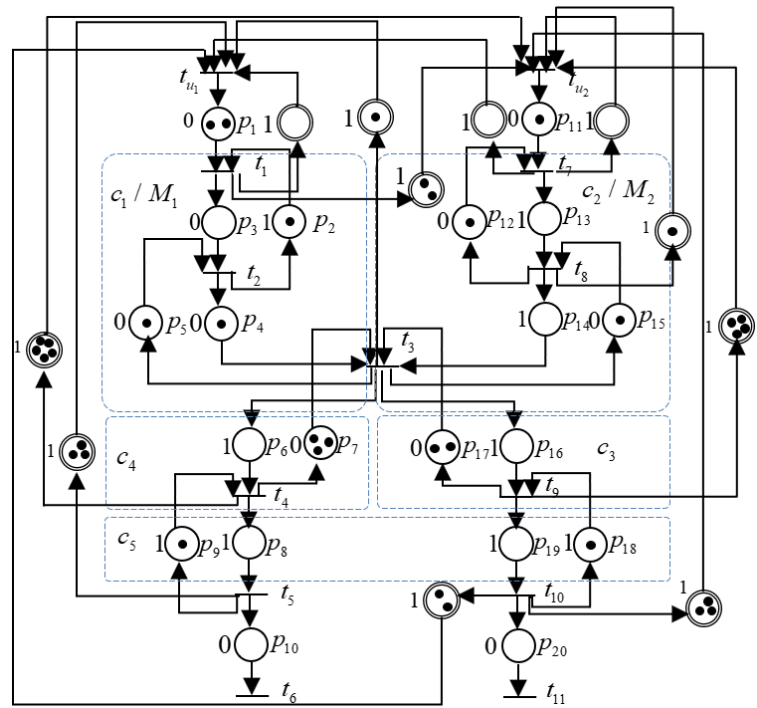

Figure 7. The TEG model of the manufacturing cell with monitor places
The controlled Timed Event Graph is illustrated in Figure 7, where the control places are represented by double circles.

\subsection{Simulation and Interpretations}

In order to highlight the control approach, the simulation of the counter functions was studied related to the dynamics of the Timed Event Graph (illustrated in Figure 8 (a) and Figure 8 (b), respectively). The comparison with the corresponding dynamic of the controlled Timed Event graph (in Figure 9 (a) and Figure 9 (b), respectively) shows that the constraints are respected. The validity of the condition in the maximum holding time within the constrained paths for the relative two counter functions $\mathrm{X} 1$ and $\mathrm{X} 7$ is checked. It is noticed that their respective events become above the event of X3 owing to the controlled model of Figure 7.

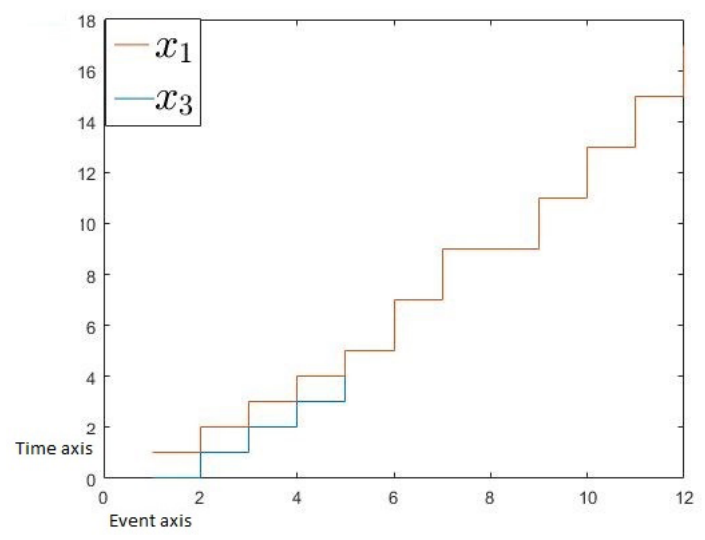

(a)

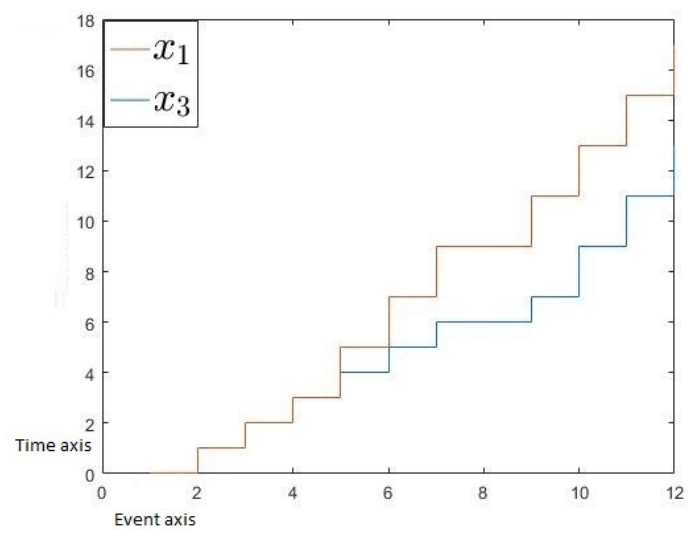

(b)

Figure 8. (a) the simulation of the dater functions $X 3$ and $\mathrm{X} 1$ before the control. (b) the simulation of the dater functions X3 and X1 after the control 


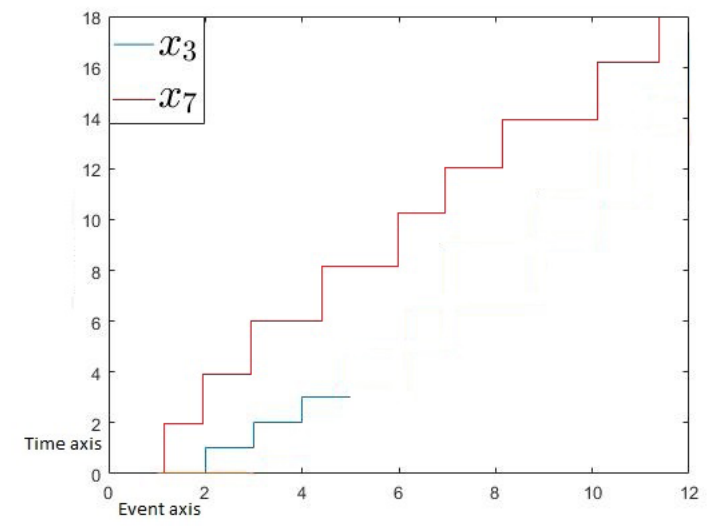

(a)

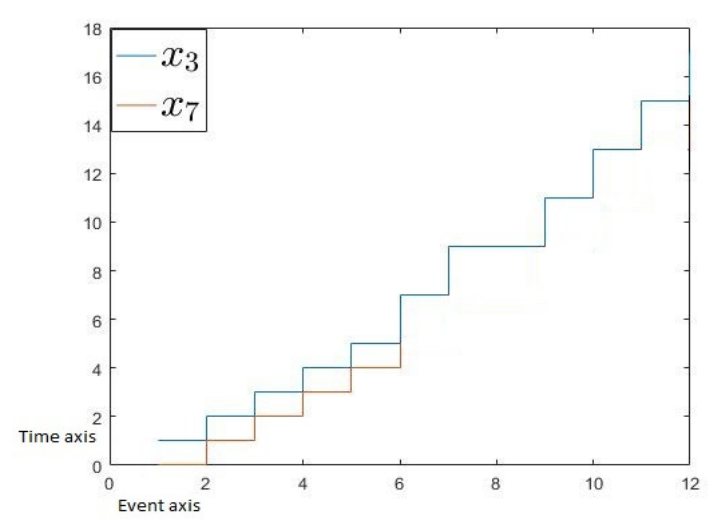

(b)

Figure 9. (a) the simulation of the dater functions $X 3$ and $\mathrm{X} 7$ before the control. (b) the simulation of the dater functions $\mathrm{X} 3$ and $\mathrm{X} 7$ after the control

\section{REFERENCES}

Addad, B., Amari, S. \& Lesage, J.-J. (2010). Analytic calculus of response time in networked automation, IEEE Transactions on Automation Science and Engineering, 7(4), 858-869.

Akian, M., Bapat, R. \& Gaubert, S. (2003). Max-plus algebras, Handbook of Linear Algebra. Chapman and Hall.

Amari, S. (2015). Feedback control for a class of discrete event systems with critical-time, International Journal of Control, 88(10), 1974-1983.

Amari, S., Demongodin, I., Loiseau, J. J. \& Martinez, C. (2012). Max-Plus Control Design for Temporal Constraints Meeting in Timed Event Graphs, IEEE Transactions on Automatic Control, 57(2), 462-467.

Atto, M. A., Martinez, C. \& Amari, S. (2011). Control of discrete event systems with respect to strict duration: Supervision of an industrial manufacturing plant, Computers \& Industrial Engineering, 61(4), 1149-1159.

\section{Conclusion}

This paper addresses the control problem of Discrete Event Systems (DES) with critical-time using the Min-Plus semiring of the dioid algebra formalism. Accordingly, a formal approach is proposed for the design of state feedback control laws to ensure the respect of temporal constraints imposed on some paths in Timed Event Graphs (TEGs). Sufficient conditions are derived for the existence of control laws satisfying the time specifications. The calculated laws are represented by a set of monitor places, and their roles are to guarantee the upper time limits for the sojourn of tokens in some paths of TEGs.

Beyond such application case, its value can be highlighted in a variety of contexts, notably for the control and validation of time constraints in urban or rail transport networks and automation systems. In a future research, it would be interesting to deal with other application cases. Work is in progress to generalize current results for more complex cases like conflicting Timed Event Graphs under time constraints. The establishing of the necessary and sufficient conditions for the existence of control laws and evaluation of the optimality of their outputs are pertinent and logical perspectives for future studies.

Baccelli, F., Cohen, G., Olsder, G. \& Quadrat, J. P. (1992). Synchronization and Linearity: An algebra for Discrete Event Systems. Wiley.

Ben Afia, N., Amari, S. \& Messaoud, H. (2019). A new formal method of control for Min-Plus linear systems subject to time constraints. In 2019 International Conference on Control, Automation and Diagnosis (ICCAD), Grenoble, France (pp. 1-6).

Bonhomme, P. (2011). Constraints Graph Based Approach for the Analysis and the Control of Time Critical Systems, International Journal of Advanced Manufacturing Technology, 57(1-4), 353-365.

Bonhomme, P. (2013). Scheduling and control of re-al-time systems based on a token player approach, Discrete Event Dynamic Systems, 23 (2), 197-209.

Cottenceau, B., Hardouin, L., Boimond, J. L. \& Ferrier, J. L. (1999). Synthesis of Greatest linear feedback for 
timed event graphs in dioid, IEEE Transactions on Automatic Control, 44(6), 1258-1262.

Dasarathy, B. (1985). Timing Constraints of RealTime Systems: Constructs for Expressing Them, Methods for Validating Them, IEEE Transactions on Software Engineering, 11(1), 80-86.

Gaubert, S. (1997). Methods and applications of $(\max ,+)$ linear algebra. In Proceedings of the 14th Annual Symposium on Theoretical Aspects of Computer Science - STACS'97, Lubeck, Germany (pp. 261-282).

Hardouin, L., Maia, C., Cottenceau, B. \& Lhommeau, M. (2010). Observer Design for $(\mathrm{Max},+)$ linear systems, IEEE Transactions on Automatic Control, 55(2), 538-543.

Heidergott, B., Olsder, G. \& van der Woude, J. (2006). Max Plus at Work: Modeling and Analysis of Synchronized Systems: A Course on Max-Plus Algebra and Its Applications. Princeton University Press.

Holloway, L. E., Krogh, B. H. \& Giua, A. (1997). A survey of Petri net methods for controlled discrete event systems, Discrete Event Dynamic Systems, 7(2), 151-190.

Houssin L., Lahaye S., \& Boimond J.-L. (2013) Control of $(\max ,+)$-linear systems minimizing delays, Discrete Event Dynamic Systems. 23(3), 261-276.

Houssin, L., Lahaye, S., \& Boimond, J.-L. (2007). Just in time control of constrained ( $\max ,+$ )-linear systems, Discrete Event Dynamic Systems, 17(2), 159-178.

Jacob, R. \& Amari, S. (2017). Observable Feedback Control of discrete processes under time constraint: Application to Cluster Tools, International Journal of Computer Integrated Manufacturing, 30(8), 880-894.

Kim, C. \& Lee, T.-E. (2016). Feedback control of cluster Tools for regulating wafer delays, IEEE Transactions on Automation Science and Engineering, 13(2), 1189-1199.

Lahaye, S., Cottenceau, B. \& Correïa, A. (2004). Commande des graphes d'événements temporisés avec contraintes de temps critique. In Conférence Internationale Francophone d'Automatique (CIFA'04), Douz, Tunisia.

Maia, C. A., Andrade, C. R. \& Hardouin, L. (2011a). On the control of max-plus linear system subject to state restriction, Automatica. vol. 47(5), 988-992.

Maia, C. A., Hardouin, L. \& Cury, J. E. R. (2013). Some results on the feedback control of max-plus linear systems under state constrains. In 52nd IEEE Conference on Decision and Control, Firenze, Italy (pp. 6992-6997).
Maia, C. A., Hardouin, L., Santos-Mendes, R. \& Loiseau. J.-J. (2011b). A super-eigenvector approach to control constrained max-plus linear systems. In: IEEE CDC-ECC, Conference on Decision and Control, European Conference on Control, Orlando, USA (pp. 136-114).

Mao, J. \& Cassandras, C. G. (2009). Optimal Control of Multi-Stage Discrete Event Systems with RealTime Constraints, IEEE Transactions on Automatic Control, 54(1), 108-123.

Mao, J. \& Cassandras, C. G. (2010). On-line Optimal Control of a Class of Discrete Event Systems with Real-Time Constraints, Discrete Event Dynamic Systems, 20(2), 187-213.

Mhalla, A., Jerbia, N., Dutilleulb, S.- C., Crayec, E. \& M. Benrejeb. (2013) Robust control strategies facing disturbances in manufacturing workshops with time constraints, Computers \& Industrial Engineering. 65(2), 268-276.

Murata, T. (1989). Petri nets: properties and applications, IEEE Proceeding, 77(4), 541-580.

Sava, A., Achour, Z., Rezg, N. (2007). Control synthesis of time Discrete Event Systems based on time controlled Petri nets, IFAC Proceedings Volumes, 40(18), 745-752.

Sava, T. A. \& Alla, H. (2006). A control synthesis approach for time discrete event systems, Mathematics and Computers in Simulation, 70, 250-265.

Tebani, K., Amari, S. \& Kara, R. (2017). Closedloop control of constrained discrete event systems: application to a networked automation system, International Journal of Advanced Manufacturing Technology, 90, 1295-1306.

Tebani, K., Amari, S. \& Kara, R. (2018). Control of Petri nets subject to strict temporal constraints using Max-Plus algebra, International Journal of Systems Science, 49(6), 1332-1344.

van den Boom, T. J. J, Lopes, G. D \& De Schutter, B. (2013). A modeling framework for model predictive scheduling using switching max-plus linear models. In 52nd IEEE Conference on Decision and Control, Florence (pp. 5456-5461).

Wang, Y., De Schutter, B., van den Boom, T. J. J. \& Ning, B. (2013). Optimal trajectory planning for trains - A pseudospectral method and a mixed integer linear programming approach, Transportation Research Part C: Emerging Technologies, 29, 97-114. 